\title{
artigo
}

Silva, A.L.; Vieira, T.S.; Ferreira, T.M.C.; Souza, L.F.; Oliveira, R.R.M.S.; Soares, A.

Caracterização de crianças com cardiopatias congênitas em um hospital no estado da Paraíba

\section{Caracterização de crianças com cardiopatias congênitas em um hospital no estado da Paraíba}

\author{
Description of children with congenital heart diseases in a hospital in the state of Paraíba \\ Caracterización de niños con enfermedades congénitas de corazón en un hospital en el estado de Paraíba
}

\begin{abstract}
RESUMO
Caracterizar o perfil clínico epidemiológico e verificar a correlação entre idade e tempo de internação de crianças com cardiopatias congênitas. Trata-se de um estudo transversal com abordagem quantitativa baseada na análise de prontuários de crianças admitidas com diagnóstico de cardiopatia congênita. A amostra foi composta por 66 prontuários. A faixa etária predominante foi a de lactentes com 54,5\%; 45,5\% apresentaram peso entre $3.500 \mathrm{~kg}$ a $10 \mathrm{~kg}$; e o comprimento de $57 \%$ da amostra encontrava-se entre 55 a $86 \mathrm{~cm}$. Houve a identificação de um estado afebril em $98,5 \%$, a frequência cardíaca em $87,7 \%$ era maior que 100 batimentos por minuto, a frequência respiratória em 92,3\% era igual ou menor que 60 incursões respiratórias por minuto, e a saturação em $78,5 \%$ dos casos era igual ou maior que $91 \%$. As cardiopatias congênitas acianóticas foram mais prevalentes. 0 estudo proporcionou a reflexão sobre as dificuldades na identificação das cardiopatias congênitas acianóticas.
\end{abstract}

DESCRITORES: Cardiopatia; Epidemiologia; Hospitalização.

\section{ABSTRACT}

Characterize the clinical epidemiological profile and to verify the correlation between age and length of hospital stay of children with congenital heart diseases in a reference hospital complex for pediatric care. This is a cross-sectional study with a quantitative approach based on the analysis of medical records of children admitted with diagnosis of congenital heart disease. The sample consisted of 66 medical records. The predominant age group was that of infants with $54.5 \% ; 45.5 \%$ had a weight between 3,500 kg to $10 \mathrm{~kg}$; and the length of $57 \%$ of the sample was between 55 and $86 \mathrm{~cm}$. There was an identification of an afebrile state in $98.5 \%$, the heart rate in $87.7 \%$ was greater than 100 beats per minute, the respiratory rate in $92.3 \%$ was equal to or less than 60 breaths per minute, and the saturation in $78.5 \%$ of cases was equal to or greater than $91 \%$. The prevalence of congenital acyanotic heart diseases. The conclusion of this study provided a reflection on the difficulties in the identification of congenital heart diseases acyanotic.

DESCRIPTORS: Heart disease; Epidemiology; Hospitalization.

\section{RESUMEN}

Caracterizar el perfil clínico epidemiológico y verificar la correlación entre la edad y la duración de la estancia hospitalaria de niños con cardiopatía congénita. Este es un estudio transversal con un enfoque cuantitativo basado en el análisis de registros médicos de niños ingresados con diagnóstico de cardiopatía congénita. La muestra consistió en 66 registros médicos. El grupo de edad predominante fue el de los lactantes con 54.5\%; El 45,5\% tenía un peso entre $3.500 \mathrm{~kg}$ y $10 \mathrm{~kg}$; y la longitud del 57\% de la muestra fue de entre 55 y $86 \mathrm{~cm}$. Se identificó un estado afebril en $98.5 \%$, la frecuencia cardíaca en $87.7 \%$ fue mayor a 100 latidos por minuto, la frecuencia respiratoria en $92.3 \%$ fue igual o menor a 60 respiraciones por minuto, y el la saturación en el $78.5 \%$ de los casos fue igual o mayor al $91 \%$. Las cardiopatías acianóticas congénitas fueron más frecuentes. El estudio proporcionó una reflexión sobre las dificultades en la identificación de cardiopatías acianóticas congénitas.

DESCRIPTORES: Cardiopatía; Epidemiología; Hospitalización.

RECEBIDO EM: 25/07/2020 APROVADO EM: 28/07/2020

\section{Amanda Lucena da Silva}

Enfermeira pelo Centro Universitário de João Pessoa.

ORCID: 0000-0001-7481-1023 
Tainelly Souza de Vieira

Enfermeira pelo Centro Universitário de João Pessoa.

ORCID: 0000-0002-1173-9009

\section{Thalys Maynnard Costa Ferreira}

Enfermeiro pela UFPB. Mestre em Enfermagem pelo PPGENF/UFPB.

ORCID: 0000-0001-8758-6937

\section{Luciana Ferreira de Souza}

Enfermeira pela UFPB. Mestra em Modelos Decisão e Saúde pelo PPGMDS/UFPB.

ORCID: 0000-0002-5998-1216

\section{Rayza Régia Medeiros dos Santos de Oliveira}

Enfermeira pela UFRN. Especialista em Enfermagem Obstétrica pela UFRN.

ORCID: 0000-0001-8725-6786

\section{Amanda Soares}

Enfermeira pela UFCG. Mestra em Saúde Pública pelo PPGSP/UEPB.

ORCID: 0000-0001-8063-4131

\section{INTRODUÇÃO}

A $s$ cardiopatias congênitas consistem em anormalidades estruturais macroscópicas do músculo cardíaco ou dos grandes vasos cardíacos com alterações funcionais significantes $^{(1)}$. A assistência perinatal interfere significativamente a redução dos fatores de risco para cardiopatia ${ }^{(2)}$.

As consequências clínicas dos defeitos cardíacos congênitos estão relacionadas a insuficiência cardíaca e hipoxemia. A insuficiência caracteriza-se por uma síndrome clínica onde o funcionamento cardíaco encontra-se inadequado às necessidades metabólicas, circulação periférica, tanto no esforço ou repouso. Já a hipoxemia, refere-se a pressão de oxigênio arterial mais baixa que o normal e uma saturação arterial reduzida, esta pode não ser evidente na avaliação clínica nos primeiros dias de vida do recém-nascido em virtude da latência do canal arterial ${ }^{(3,4,5)}$.

Em 2010 foram notificados ao Sistema Nacional de Nascidos Vivos, 1.377 casos de nascidos com cardiopatias congênitas, o que representa cerca de 5,3\% do estimado para o Brasil. Em 2014 foi considerada como a terceira maior causa de mortalidade neonatal ${ }^{(6,7)}$.

$\mathrm{Na}$ Paraíba, a assistência à saúde da criança com cardiopatia congênita é ofertada nas maternidades estaduais. Essa assistência foi garantida por meio de uma parceria entre os Estados da Paraíba e Pernambuco, no ano de 2011, objetivando facilitar o tratamento de crianças carentes, portadoras de doença cardíaca ${ }^{(8)}$.

Os avanços existentes $\mathrm{e}$ inserção de programas de apoio à criança com cardiopatia congênita, não garantem a redução da mortalidade neonatal por cardiopatia congênita, sendo a população de classe socioeconômica baixa com grande representatividade desta porcentagem ${ }^{(9)}$.

Diante disso, a identificação precoce das características clínicas e epidemiológicas podem promover o prognóstico favorável de crianças com cardiopatia congênita, sendo capaz de reduzir as taxas de morbimortalidade.

Para tanto, o presente estudo objetiva caracterizar o perfil clínico epidemiológico e verificar a correlação entre idade e tempo de internação de crianças com cardiopatias congênitas de um complexo hospitalar referência para assistência pediátrica.

\section{MÉTODOS}

Trata-se de um estudo transversal com abordagem quantitativa baseada na análise de prontuários de crianças ad- mitidas com diagnóstico de cardiopatia congênita. Foi realizada durante os meses de setembro a outubro de 2018 no serviço de arquivo médico do Complexo de Pediatria Arlinda Marques localizado da cidade de João Pessoa/PB.

Em 2017, ano utilizado como base para esse estudo, foram identificadas em 108 prontuários de crianças admitidas com cardiopatia congênita e a amostra totalizou-se em 66 prontuários.

Para a análise estatística descritiva foi utilizado o Statistical Package for the Social Sciences versão 24.0 e foram calculadas a média e equivalente porcentagem. Para a avaliação da relação entre a idade e o tempo de hospitalização foi calculado o desvio-padrão e variância destes e utilizado o teste de correlação de Pearson $(p<0,05)$. Este estudo foi aprovado pelo Comitê de Ética e Pesquisa do Centro Universitário de João Pessoa com número de CAAE 95404518.3.0000.5176.

\section{RESULTADOS}

A amostra foi constituída por 66 prontuários de crianças com diagnóstico de cardiopatia congênita dos quais $54,5 \%$ (36) dos pacientes eram do sexo masculino. Em 45,5\% (30) dos casos o peso dos pacientes era maior que 3.500 a menor ou igual a $10 \mathrm{~kg}$, e em $57 \%$ 


\section{artigo}

Silva, A.L.; Vieira, T.S.; Ferreira, T.M.C.; Souza, L.F.; Oliveira, R.R.M.S.; Soares, A.

Caracterização de crianças com cardiopatias congênitas em um hospital no estado da Paraíba

(38) o comprimento variava de 55 a 86 cm (Tabela 1).

De acordo com o estudo a maioria dos pacientes não apresentava temperatura elevada, caracterizando um estado afebril em 98,5\% (65), a frequência cardíaca em $87,7 \%$ (58) era maior que 100 batimentos por minuto, a frequência respiratória em 92,3\% (61) era igual ou menor que 60 incursões por minuto, e a saturação em 78,5\% (51) dos casos era igual ou maior que $91 \%$.

Os tipos de cardiopatias congênitas mais frequentes no ano de 2017 foram: a persistência do canal arterial com 23,4\% (15); o defeito do septo atrial com $18,8 \%$ (12); e o defeito do septo ventricular com 17,2\% (11) (Tabela 1).

A idade média das crianças com diagnóstico de cardiopatia congênita foi de 2,5 $\pm 2,8$ anos. O tempo de hospitalização foi de 11,12 \pm 8,6 dias, com mínimo de um e no máximo 40 dias (tabela 2). No teste de significância de Pearson $(\mathrm{p}<0,05)$ podese observar que não teve correlação entre a idade da criança e o tempo de hospitalização evidenciado pelo $\mathrm{p}=0,677$.

No que concerne ao tipo de desfecho da internação de crianças com cardiopatias congênitas admitidas no ano de 2017 o mais prevalente foi o tratamento cirúrgico, equivalente à $69,7 \%$ (46) cirurgias realizadas (Tabela 3 ).

\section{DISCUSSÃO}

Para Silva et al. ${ }^{(10)}$, cerca de dez em

Tabela 1: Diagnósticos de cardiopatias congênitas em uma unidade hospitalar do estado da Paraíba.

\section{TIPO DE CARDIOPATIA}

Persistência do canal arterial

Defeito do septo atrial

Defeito do septo ventricular

Tetralogia de Fallot

Defeito do septo atrioventricular

Outras

Fonte: Dados da pesquisa, 2018

Tabela 2: Valores médios, desvio-padrão e intervalo de confiança da idade e tempo de hospitalização de crianças com cardiopatias congênitas admitidas em 2017.

\begin{tabular}{lcc}
\multicolumn{1}{c}{ VARIÁVEIS } & Média \pm DP & IC95\% \\
Idade (anos) & $2,5 \pm 2,8$ & $0,008-9,7$ \\
Tempo de hospitalização (dias) & $11,12 \pm 8,6$ & $1-40$ \\
DP: desvio padrão; I IC95\%: intervalo de confiança de 95\%; p: valor de significância. Fonte: Dados da pesquisa, 2018.
\end{tabular}

Tabela 3: Desfecho da internação de crianças com cardiopatias congênitas admitidas em 2017.

$\begin{array}{lc}\text { DESFECHO } & \mathbf{N}(\%) \\ \text { Óbito } & 6(9,1) \\ \text { Cirurgia } & 46(69,7) \\ \text { Cirurgia remarcada } & 4(6,1) \\ \text { Tratamento Clínico } & 9(13,6) \\ \text { Não informado } & 1(1,5) \\ \text { Fonte: Dados da pesquisa, 2018. } & \end{array}$

cada mil recém-nascidos vivos são afetados por algum tipo de malformação congênita. Essas malformações apresentam uma ampla sintomatologia clínica.

Neste estudo, verificou-se que a frequência cardíaca estava acima de 100 batimentos por minuto em $87,7 \%$ dos casos. Considera-se a frequência cardíaca apresentada normal para criança, justificado pelo seu metabolismo acelerado. Compreende-se que o público estudado era em sua maioria, lactentes (54,5\%), o que fortalece a relação com os resultados apresentados. Portanto, essa associação aplicase também a frequência respiratória e temperatura ${ }^{(1)}$.

A maioria dos pacientes apresentaram clinicamente afebril, normocárdico, eupneico e com saturação de $\mathrm{O} 2$ adequada. Supõem-se que esses achados possuem ligações com os tipos de cardiopatias prevalentes no estudo.

Para a compreensão dos resultados encontrados, faz-se necessário entender que as cardiopatias congênitas do tipo acianóticas são caracterizadas pelo não aparecimento da cianose previamente, pois não há obstrução de sangue venoso na circulação sistêmica. Desse modo, as consequências clínicas apresentam-se leves e tardias quando comparadas as cianóticas, justificando a estabilidade nos parâmetros da amostra deste estudo ${ }^{(11)}$. A tetralogia de Fallot apresentou prevalência de $14,1 \%{ }^{(9)}$ considerada uma cardiopatia congênita cianótica em virtude da oxigenação insuficiente ${ }^{(1)}$.

Levando em consideração a predominância da clínica estabilizada diretamente relacionada a prevalência de cardiopatias acianóticas e de lactentes, justifica-se a relação supracitada a partir das características anatômicas e fisiológicas no organismo do lactente, pois nessa faixa etária o sistema nervoso atua com uma efetiva vasoconstrição, considerada mais eficiente que em adultos, minimizando os efeitos da hipoxemia. Sendo assim, acatadas como parte do processo fisiológico de adaptação ao meio extrauterino, não evidencia alterações clínicas imediatas para a criança, 
dificultando a identificação prévia das malformações cardíacas ${ }^{(12)}$.

A identificação das características clínicas das cardiopatias congênitas é intrínseca ao prognóstico da criança hospitalizada, assim como a promoção de uma assistência segura e continua. Para isso, há uma necessidade de fortalecimento da assistência executada por profissionais atuantes na saúde da criança, realizando uma avaliação clínica contínua desde o período intrauterino, perdurando ainda no extrauterino ${ }^{(13)}$.

Referente a assistência ofertada é importante ressaltar esta não é considerada fator determinante do tempo de internação de uma criança diagnosticada com cardiopatia congênita, há também associação a diversos fatores como recursos que estão disponíveis na instituição, idade, estado nutricional, condição clínica prévia, dor e até a escolaridade ${ }^{(14)}$.

A associação entre a idade de crianças com cardiopatia congênitas e o tempo de hospitalização não teve significância estatística $(p<0,05)$, indicando que outros fatores estão intrinsicamente envolvidos.

No tocante ao tratamento da cardiopatia, este dependerá do tipo e da gravidade, em cardiopatias leves há elevada possibilidade de cura sem intervenção medicamentosa ou cirúrgica. Em outros casos podem ser necessários tratamentos medicamentosos ou cirurgia cardíaca ${ }^{(15,16)}$.

Em estudo de realizado entre os anos de 2008 a 2013 identificou-se que a taxa de mortalidade infantil por cardiopatias congênitas tem apresentado tendência a diminuir devido às melhores condições do pré-natal e a possibilidade de acesso ao diagnóstico e tratamento precoce ${ }^{(17)}$.

\section{CONCLUSÃO}

Perante os resultados encontrados, limitações durante o processo de coleta foram notadas, como a quantidade de prontuários da amostra inferior ao esperado, o que pode ter contribuído para o achado da correlação não significativa entre as variáveis tempo de hospitalização e idade. A justificativa relaciona-se às perdas devido à ausência de preenchimento de dados no prontuário, deixando lacunas sobre a avaliação realizada e a assistência prestada.

Diante do exposto, esse estudo proporcionou a reflexão sobre as dificuldades na identificação das cardiopatias congênitas, especificamente as acianóticas, além da prevalência em lactentes, faixa etária a qual ainda podem ocorrer alterações fisiológicas nas crianças. 匹

\section{REFERÊNCIAS}

1. Belo WA, Oselame GB, Neves EB. Perfil clínico-hospitalar de crianças com cardiopatia congênita. Cader Saúde Coletiva. 2016; 24(2): 216-220.

2. Pinto CP, Westphal F, Abrahão AR. Fatores de riscos materno associados à cardiopatia congênita. J Health Sci Inst. 2018; 36(1): 34-08.

3. Honckenberry MJ, Wilson D. Wong: Fundamentos de enfermagem pediátrica. 10ª ed. Rio de Janeiro: Elsevier, 2014.

4. Magalhães CC, Jr CVS, Colombo FMC, Nobre F, Fonseca FAH, Ferreira JFM. Tratado de Cardiologia SOCESP. $3^{\circ}$ ed, Rio de Janeiro: Manole, 2015.

5. Lacerda LF, Ferreira ALC, Lisboa CB, Lúcio IML, Batista JCL, Melo LO. Triagem neonatal de cardiopatias congênitas: percepção dos profissionais de saúde do alojamento conjunto. Rev Enferm UFPE online. 2016; 10(7): 2420-7.

6. Pinto Júnior VC, Branco KMPC, Cavalcante RC, Carvalho Junior W, Lima JRC, Freitas SM, et al. Epidemiology of congenital heart disease in Brazil. Brazilian Journal of Cardiovascular Surgery. 2015; 30(2): 219-224.

7. BRASIL. Portaria n 1.727, de 11 de julho de 2017. Aprova o Plano Nacional de Assistência à Criança com Cardiopatia Congênita. 2017.

8. CIRCOR. Círculo do Coração. Quem somos? 2019 [Citado em 4 nov 2019]. Disponivel em: https://www.c-online.med.br/circor-2019-quem-somos/

9. Souza ATS, Amaral LRS, Pereira MS, Freire VS, Alves, AS, Araújo $A P M$, et al. Estado vacinal de adolescentes de uma unidade básica de saúde. Ver Eletr Acer Saúde. 2020; 12(6): e3059.
10. Silva ACS, Souza, TP, Santos El, Knupp VMAO. Configuração do serviço de saúde no município de rio das ostras frente ao diagnóstico e tratamento de cardiopatia congênita. Rev Enfer UFJF. 2016; 2(2).

11. Araújo JSS, Régis CT, Gomes RGS, Silva CS, Abath CMB, Mourato $F A$, et al. Cardiopatia congênita no nordeste brasileiro: 10 anos consecutivos registrados no estado da Paraíba, Brasil. Rev Bras Cardiol, 2014; 27(1): 509-15.

12. Aehlert B. PALS: Suporte Avançado de Vida em Pediatria: Guia de estudo. $3^{\text {a }}$ ed. Rio de Janeiro: Elsevier. 2014.

13. Medeiros AL, Freitas TB, Araújo JSS, Mattos SS. Oximetria de pulso em triagem de cardiopatias congênitas: conhecimento e atuação do enfermeiro. Cogitare Enfer. 2015; 20(3).

14. Silva AMN, Souza EFD, Barbosa TLA, Silva CSO, Gomes LMX. Fatores que contribuem para o tempo de internação prolongada no ambiente hospitalar. J. res.: fundam. Care. Online. 2014; 6(4):1590-1600.

15. Jesus VS, Nascimento AM, Miranda RA, Lima JS, Tyll MAG, Veríssimo AOL. Fila de espera para tratamento de pacientes com cardiopatia congênita: Retrato de um centro de referência amazônico. Inter Jour of Cardiovasc Sciences. 2018; 31(4): 374-382.

16. Saliba A, Figueiredo ACV, Baroneza JE, Afiune JY, Taylor AP, Oliveira SF, Mazzeu JF. Genetic and genomics in congenital heart disease: a clinical review. J Pedia, 2020; 96(3): 279-288.

17. Braga DC, Saccol MP, Conte TA, Goldmeier R, Pereira RW. Evolução da mortalidade por cardiopatias congênitas no Brasil - um estudo ecológico. Rev Instit Ciências Saúde. 2017; 35(2):105-7. 